\title{
Malignización del liquen plano oral
}

\author{
Cerero-Lapiedra R*
}

\section{RESUMEN}

La complicación más importante del Liquen Plano Oral (LPO), es el desarrollo de un carcinoma oral de células escamosas (COCE). Desde 1910 en que se comunicó el primer caso en una paciente con LP, se han publicado numerosos casos, y series de casos de malignización, que nos aportan cifras que varían entre el 0 y el $12,5 \%$ de LPO malignizados, con periodos de seguimiento también muy variables, que oscilan entre 0,5 y 20 años. Pero estos estudios han sido refutados por un grupo de autores, que se cuestionan los criterios diagnósticos utilizados.

Por todo ello, el potencial maligno del liquen plano oral, es un tema actualmente muy controvertido en Medicina Oral. Se realiza una revisión crítica de la literatura más actual sobre los conceptos clave, implicados en esta controversia.

Palabras clave: Liquen plano oral, Malignización, Diagnóstico, Seguimiento.

\section{SUMMARY}

The most important complication of oral lichen planus (OLP), is the development of oral squamous cell carcinoma (OSCC).

From 1910 when was reported the first case in a woman suffered OLP, many cases and cases series have been reported with values from 0 to $12,5 \%$, and with follow-up ranking from 0,5 to 20 years. But many of this cases not had been accepted by some authors who disagree with the diagnostic criteria employed.

By all previously cited, the cancerization of OLP is a very controversial topic in Oral Medicine. It has been performed a critical review of the recent literature about it.

Key words: Oral lichen planus, Malignancy, Dianosis, Follow-up.

Fecha de recepción: Diciembre 2007.

Aceptado para publicación: Enero 2008.

* Profesora Titular de Patología Médica Bucal. UCM.

Cerero-Lapiedra R. Malignización del liquen plano oral. Av. Odontoestomatol 2008; 24 (1): 97-103.

\section{INTRODUCCIÓN}

El liquen plano es una enfermedad inflamatoria y de naturaleza autoinmune que afecta al epitelio escamoso estratificado, y que se manifiesta en piel y mucosas. Mientras que el liquen de la piel es autolimitado, las lesiones orales son cróni- cas, potencialmente premalignas y, en pocos casos, entre un 6 y un $11 \%$, remiten espontáneamente.

El liquen plano oral (LPO) se presenta con más frecuencia a partir de la quinta década de la vida, y es hasta tres veces más frecuente en mujeres. 
Las lesiones orales se caracterizan por su polimorfismo. Hay varios tipos de lesiones: reticular (típica), en placa, eritematosa (atrófica), erosiva, y en forma de gingivitis descamativa.

Actualmente el tratamiento es sintomático, y principalmente basado en el empleo de corticoides, tanto por vía tópica como sistémica, e incluso mediante inyección perilesional.

Hay algunos factores tanto sistémicos como locales que se relacionan con una mayor agresividad y/o resistencia al tratamiento: infección por el virus de la Hepatitis C, diabetes mellitus, factores psicológicos, reacciones a diversos materiales dentales una mala odontología conservadora y la presencia de placa dental (1).

\section{SERIES PUBLICADAS DE CASOS DE LPO MALIGNIZADOS}

Desde 1910 en que se comunicó el primer caso de aparición de COCE en un paciente con LPO, se han publicado numerosas series de casos, que nos aportan cifras de transformación maligna muy variables. Este aparente incremento del riesgo de que aparezca un COCE en pacientes con LPO, respecto a las personas que no lo padecen, parece ser para algunos autores independiente de tipo clínico y de la terapia administrada (2). Para otros, sin embargo, las formas clínicas erosiva y en placa tendrían un potencial de malignización mayor, pero esta hipótesis no ha podido ser demostrada. Además, no parece que los factores de riesgo implicados en el cáncer oral, como son el tabaco y el alcohol, jueguen un papel en la transformación del LPO (3).

Hemos seleccionado 27 de todas las series publicadas desde 1968 hasta 2007, entre las que se referían a un mayor número de casos y aportaban más datos de diagnóstico. Entre todas ellas, el número de casos totales de LPO es de 9.837, y la media de casos que se han malignizado, de un 1,5\%, lo que resulta realmente una cifra pequeña. El tiempo de seguimiento de los pacientes es muy variable, así como el tiempo desde el diagnóstico de LPoy la aparición del COCE (Tablas 1 y 2).

TABLA 1.- SERIES PUBLICADAS DESDE 1968 HASTA 1993

\begin{tabular}{|c|c|c|c|c|c|c|c|}
\hline Autor & Año & País & $\begin{array}{c}\mathrm{N}^{\circ} \\
\text { pacientes } \\
\text { liquen }\end{array}$ & $\begin{array}{c}\mathrm{N}^{\circ} \text { casos } \\
\text { malignizados }\end{array}$ & $\%$ & $\begin{array}{l}\text { Años } \\
\text { obser- } \\
\text { vación }\end{array}$ & $\begin{array}{c}\% \text { casos } \\
\text { malignizados } \\
\text { por año }\end{array}$ \\
\hline Andreasen JO & 1968 & Dinamarca & 115 & 0 & 0 & 10 & 0 \\
\hline Shklar G & 1972 & USA & 600 & 3 & 0,5 & - & - \\
\hline Grinspan D & 1972 & Argentina & 237 & 16 & 6,3 & - & \\
\hline Fulling HJ & 1973 & Dinamarca & 225 & 1 & 0,4 & 3,6 & 0,12 \\
\hline Kóvesi and Banoczy & 1973 & Hungría & 274 & 1 & 0,4 & - & - \\
\hline Silverman et al & 1985 & USA & 570 & 7 & 1,2 & 5,6 & 0,22 \\
\hline Murti et al & 1986 & India & 702 & 3 & 0,4 & 5,1 & 0,08 \\
\hline Holmstrup et al & 1988 & Dinamarca & 611 & 9 & 1,5 & 7,5 & 0,20 \\
\hline Salem G & 1989 & Arabia Saudí & 72 & 4 & 5,6 & 3,2 & 1,74 \\
\hline Vincent S & 1990 & USA & 100 & 0 & 0 & 0,7 & 0 \\
\hline Silverman et al & 1991 & USA & 214 & 5 & 2,3 & 7,5 & 0,31 \\
\hline Voute et al & 1992 & Holanda & 113 & 3 & 2,7 & 7,8 & 0,34 \\
\hline Moncarz et al & 1993 & Israel & 280 & 6 & 2,1 & - & - \\
\hline Barnard et al & 1993 & Reino Unido & 241 & 8 & 3,3 & - & - \\
\hline
\end{tabular}




\section{TABLA 2.- SERIES PUBLICADAS DESDE 1993 HASTA 200}

\begin{tabular}{|c|c|c|c|c|c|c|c|}
\hline Autor & Año & País & $\begin{array}{c}\mathrm{N}^{\circ} \\
\text { pacientes } \\
\text { liquen }\end{array}$ & $\begin{array}{c}\mathrm{N}^{\circ} \text { casos } \\
\text { malignizados }\end{array}$ & $\%$ & $\begin{array}{l}\text { Años } \\
\text { obser- } \\
\text { vación }\end{array}$ & $\begin{array}{c}\% \text { casos } \\
\text { malignizados } \\
\text { por año }\end{array}$ \\
\hline Brown et al & 1993 & USA & 193 & 0 & 0 & 2,9 & \\
\hline Gorsky et al & 1996 & Israel & 157 & 2 & 1,3 & 1,5 & 0,85 \\
\hline Markopoulos et al & 1997 & Grecia & 326 & 4 & 1,3 & 4,8 & 0,26 \\
\hline Silverman and Bahl & 1997 & USA & 95 & 3 & 3,2 & 6,1 & 0,52 \\
\hline Lo Muzio et al & 1998 & Italia & 263 & 13 & 4,9 & 5,7 & 0,86 \\
\hline Rajentheran et al & 1999 & Reino Unido & 832 & 7 & 0,8 & 11 & 0,07 \\
\hline Mignogna et al, & 2001 & Italia & 502 & 18 & 3,6 & - & - \\
\hline Eisen & 2002 & USA & 723 & 6 & 0,8 & 4,5 & 0,18 \\
\hline Van der Meij et al & 2003 & Holanda & 173 & 3 & 1,7 & 2,7 & 0,65 \\
\hline Rödstrom et al & 2004 & Suiza & 1028 & 5 & 0,5 & 6,8 & 0,07 \\
\hline Gandolfo et al & 2004 & Italia & 402 & 9 & & 4,9 & \\
\hline Xue et al & 2005 & China & 674 & 4 & 0,5 & - & - \\
\hline Bornstein M & 2006 & Suiza & 145 & 4 & 2,8 & 3,7 & 0,35 \\
\hline Hsue et al & 2007 & Taiwan & 143 & 3 & 2,1 & 1,1 & - \\
\hline
\end{tabular}

Modificado de van der Meij et al (Oral Oncol 2007: 43742-8).

El problema de la disparidad de resultados entre unas series y otras, podría deberse a que no tienen unos criterios equivalentes en la selección, identificación y seguimiento de los pacientes.

En estas series podemos observar varias diferencias fundamentales:

- Diferencias en los criterios diagnósticos: el diagnóstico de LPO se realiza de manera muy diferente, en algunos estudios es únicamente clínico, mientra que en otros también incluye el estudio histopatológico. Los criterios tanto clínicos como histopatológicos empleados, generalmente no se especifican.

- Diferencias en los datos aportados: en pocos estudios encontramos el dato de la ratio de trasformación media anual. En los estudios en que se halla, comprobamos que oscila desde 0 a 1,74. Este dato es muy interesante ya que ofrece información real respecto a la población general.

- Diferencias en los criterios de inclusión: se incluyen pacientes consumidores y no consumidores de alcohol y tabaco indistintamente, que padecen o no diferentes enfermedades sistémicas, con lesiones de tiempo de evolución variable, y que han sido tratadas con distintas pautas.
- Diferencias en el tiempo de evolución: el seguimiento desde el diagnóstico tanto de los casos malignizados como del restote los casos, no son iguales. El tiempo medio de seguimiento de los pacientes con LPO, es muy variable, desde 0.5 hasta 20 años. No es comparable la aparición de un COCE en un liquen de varios años de evolución, como en uno recién diagnosticado, en cuyo casos podría tratarse de enfermedades concomitantes (2).

- Diferencias en los datos de localización: respecto a los casos que han sufrido malignización, no aparece siempre el dato de la localización de las lesiones previas de LPO.

Existiendo esta gran variabilidad de criterios, la realización de un metaanálisis es poco viable ya que no existe homogeneidad de criterios. Por todo ello, no es posible extraer conclusiones con suficiente evidencia científica.

\section{PROBLEMAS EN EL DIAGNÓSTICO DE LPO}

El mayor problema que se puede observar a la hora de homogeneizar las series, es el de los criterios diagnósticos de LPO que se emplean. El diagnóstico de LPO se debe confirmar con una biopsia, aunque hay 
autores que opinan que en los casos en los que las lesiones orales sean típicas o si existen lesiones concomitantes en piel, puede ser suficiente con el diagnóstico clínico (4).

En 1978, la OMS formuló una definición tanto clínica como histopatológica de LPO, y desde entonces se ha usado como criterio de inclusión en los diferentes estudios sin que se haya revisado(5). Recientemente algunos autores han propuesto unos criterios diagnósticos basados en la anterior definición. Esta nueva propuesta ha surgido a raíz de los resultados poco satisfactorios obtenidos en los estudios sobre concordancia diagnóstica en LPO tanto entre clínicos como entre histopatólogos (6).

Los criterios propuestos serían los siguientes:

- Criterios clínicos:

- Presencia de lesiones bilaterales más o menos simétricas.

- Presencia de lesiones lineales blancas-grisáceas que siguen un patrón reticular.

- Se aceptan las lesiones erosivas, atróficas ampollares y en placa como subtipos, en presencia de lesiones reticulares en cualquier otra localización.

- El resto de lesiones, que parezcan LPO pero no completen los criterios anteriormente mencionados, se denominan: "clínicamente compatible con".

- Criterios histopatológicos:

- Signos de licuefacción-degeneración de la capa de células basales.

- Ausencia de displasia.

- Si los signos histopatológicos son menos obvios, se denomina "histológicamente compatible con".

Liquen plano. El diagnóstico de liquen plano requiere que se cumplan los criterios tanto clínicos como histopatológicos. Independientemente de todo ello, cuando el liquen se manifiesta como Gingivitis Descamativa el diagnóstico es más difícil pero el análisis por inmunofluorescencia directa de una zona adyacente, donde se encuentra fibrinógeno en un patrón lineal en la zona de la membrana basal, puede contribuir a facilitar la identificación del LPO (4).

\section{LIQUEN PLANO VS LESIÓN LIQUIENOIDE}

Clásicamente se reservaba el término lesión o reacción liquenoide para aquellas lesiones de la cavidad oral con una etiología determinada, por ejemplo una reacción adversa a algún tipo de material de restauración dental, o la ingesta de algún determinado fármaco, y cuya clínica e histopatología era similar a la del liquen plano oral (Eisen, 2005).

El método más eficaz de diagnosticar la reacción liquenoide oral (RLO) sería la constatación de que la patología remite cuando se retira el fármaco o la sustancia responsable, y vuelve a aparecer si se repite la situación. La realidad no es así, puesto que en muchas ocasiones no remiten las lesiones hasta meses después de la retirada del fármaco y, de la misma forma la aparición de la RLO no aparece hasta un tiempo después del comienzo de la ingesta. Por todo ello, el diagnóstico y la relación causa-efecto, en muchas ocasiones resulta difícil de establecer. Los fármacos implicados en estas RLO son los antiinflamatorios no esteroideos, betabloqueantes, metildopa, penicilamina y algunos antibióticos. Se han mencionado otros muchos, pero se trata de casos aislados.

Respecto a los materiales de restauración dental, todos ellos han sido implicados como causantes de reacciones liquenoides. Actualmente se discute sobre la utilidad de retirar las restauraciones de amalgama en los pacientes con LPO. Existen tendencias contrapuestas, pero tanto en los casos en los que existan pruebas alérgicas positivas a alguno de los componentes de la amalgama, como cuando las lesiones estén localizadas en contacto con alguna restauración, parece aceptado que se deben retirar dichas restauraciones y que generalmente las lesiones remiten (4).

Recientemente existe cierta controversia sobre el término lesión/reacción liquenoide oral y, según algunos autores, se denominaría así a los casos en los que el diagnóstico de liquen no está totalmente claro (6).

El término "lesión oral liquenoide" se usaría en los siguientes supuestos:

1. Cuando existe un diagnóstico clínico de LPO pero el diagnóstico histológico es solamente compatible con LPO. 
2. Cuando por el contrario, el diagnóstico histológico es de certeza pero clínicamente es solamente compatible con LPO.

3. Tanto clínica como histológicamente solamente compatible con LPO.

Por tanto, el término lesión liquenoide se reservaría para las siguientes circunstancias:

1. Lesiones típicas clínicamente pero "compatibles "histológicamente.

2. Lesione típicas histológicamente pero "compatibles" desde un punto de vista clínico.

3. Lesiones únicamente "compatibles" desde ambos puntos de vista.

\section{DISPLASIA LIQUENOIDE}

Para algunos autores, la gran variabilidad en las series de casos de malignización de LPO, se debería a un diagnóstico erróneo de LPO. Krutchkoff y Eisembrerg describen una nueva entidad: Displasia liquenoide, que sería el auténtico diagnóstico inicial de muchos de los casos malignizados.

La Displasia liquenoide se describiría como una condición precancerosa con características liquenoides, siendo la presencia de displasia, la característica principal que diferencia ambas entidades (7).

Estos autores, proponen unos criterios diagnósticos de LPO, para aceptar los casos de malignización publicados (8).

A. Diagnóstico Original: Diagnóstico clínico y por lo menos 2 de las siguientes características histológicas.

1. Hiperqueratosis o paraqueratosis.

2. "Dientes de sierra".

3. Infiltrado superficial de linfocitos.

4. Liquefacción de las células basales.

B. Historia y seguimiento:

1. Características clínicas precisas, de la evolución, localización, tratamiento...

2. Seguimiento mínimo durante 2 años.

\section{Exposición a tabaco: Debe ser documentada.}

Recientemente se han publicado nuevas series aplicando criterios diagnósticos estrictos, y se demuestra estadísticamente un aumento de riesgo de padecer carcinoma de células escamosas en los pacientes con LPO. Estos estudios no son capaces de identificar los factores que modifican ese riesgo en determinado sujeto con $\operatorname{LPO}(2,9,10)$.

\section{SEGUIMIENTO DE LOS PACIENTES CON LIQUEN PLANO ORAL}

De todo lo anteriormente planteado, se deriva una importante cuestión. ¿Hasta que punto están justificadas las revisiones en estos pacientes? Hay disparidad de opiniones respecto a su necesidad, utilidad y protocolo. Autores como Mignogna, consideran imprescindible un seguimiento exhaustivo, considerando que así se podría diagnosticar precozmente la transformación maligna de las lesiones de LPO $(11,12)$.

Proponen el siguiente protocolo de seguimiento:

- Seguimiento 3 veces /año o más.

- Factores modificadores exhaustivamente controlados.

- Examinar cuidadosamente en cada visita la morfología y distribución de las lesiones.

- Áreas queratóticas: observar cuidadosamente si existe pérdida local de la homogeneidad de las mismas (Fig 1).

- Áreas rojas (atróficas/erosivas) sintomáticas: tratamiento precoz para diferenciar de otras entidades en base a la respuesta al tratamiento (Fig 2).

- Áreas rojas asintomáticas: prestar atención a la homogeneidad de la lesión, presencia de estrías y cualquier signo que oriente sobre si es o no una lesión de LPO (Fig 3).

Sin embargo, otro grupo de autores como consideran poco útil el seguimiento ya que consideran en primer lugar que no es posible identificar a los pacientes con un elevado riesgo de desarrollo de cáncer y además, que no es posible reducir la morbilidad y mortalidad del cáncer oral con un programa de visitas frecuentes (13). 


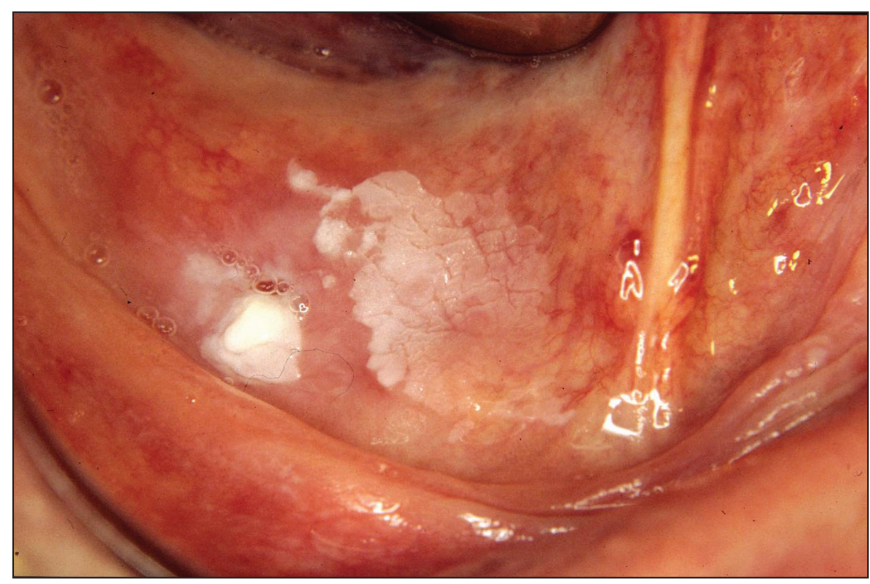

Fig. 1. Áreas queratóticas de LPO.

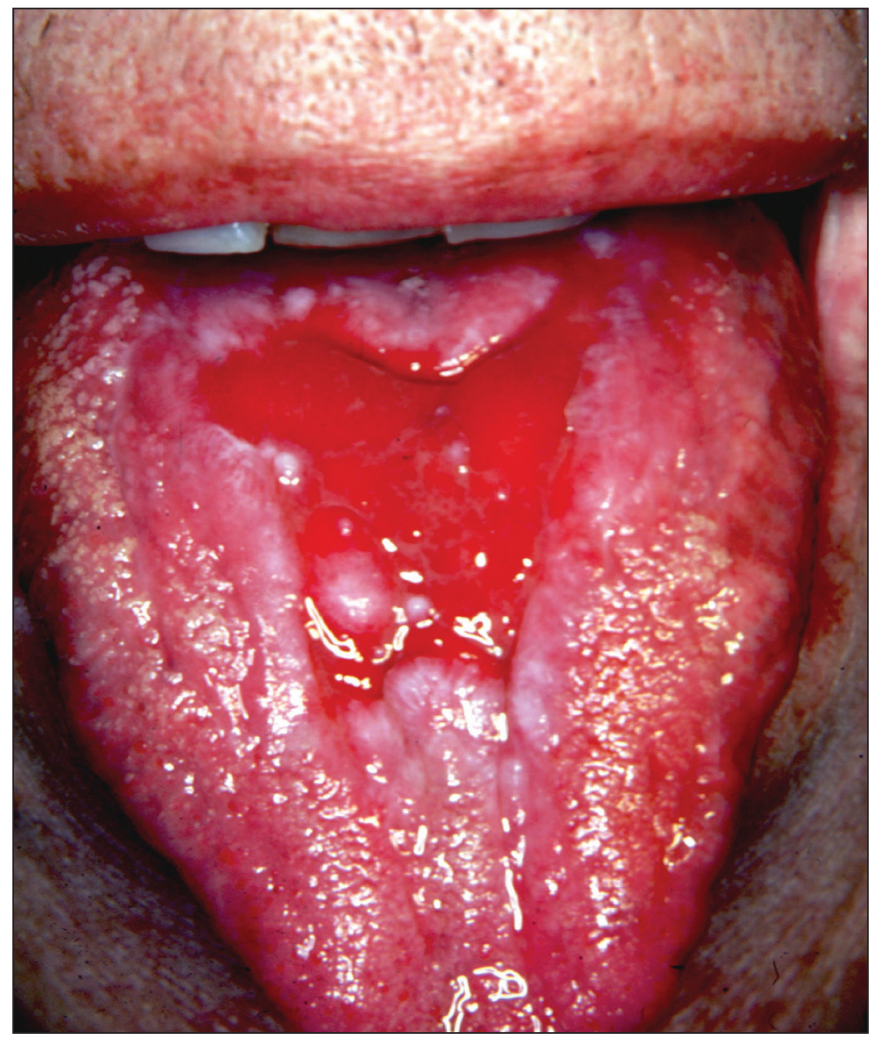

Fig. 2. LPO atrófico erosivo sintomático.

\section{CONCLUSIONES}

1. Es importante que exista un consenso para definir las características que permiten hacer un diagnóstico de LPO, y si entre ellas está la presencia o no de displasia epitelial.

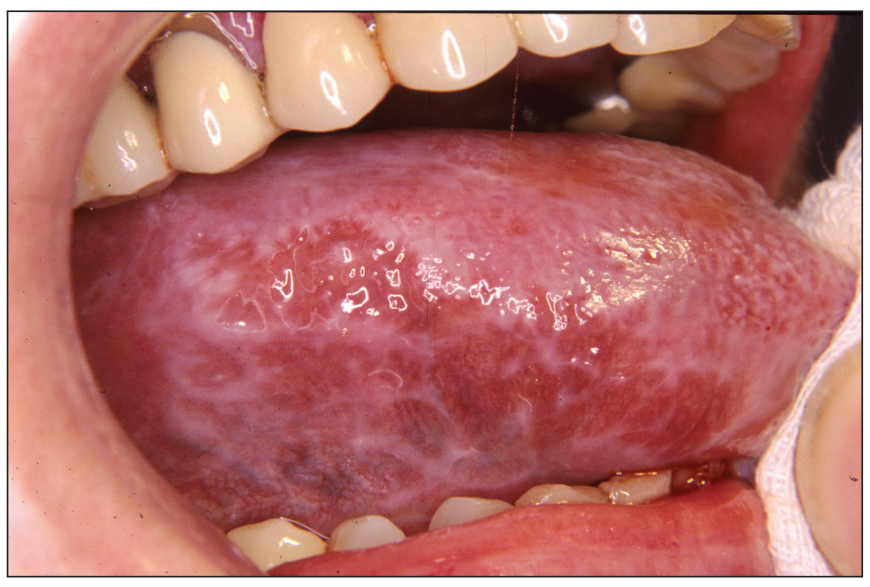

Fig. 3. Pérdida de homogeneidad de lesiones de LPO.

2. Serían necesarios estudios homogéneos para valorar realmente la capacidad de malignización del LPO.

3. Con todo ello, se debería establecer un protocolo de seguimiento de estas lesiones que dependería de los anteriores apartados.

\section{BIBLIOGRAFÍA}

1. Sumairi B, Kumar S, Zain R. Oral lichen planus and lichenoid reactions: etiopathogenesis, diagnosis, management and malignant transformation. Journal of Oral Science 2007; 49: 89-106.

2. Gandolfo S, Richiardi L, Carrozzo M et al. Risk Of oral squamous cell carcinoma in 402 patients with oral lichen planus: a follow-up study in an Italian population. Oral Oncol 2004;40:562-70.

3. van der Meij EH, Schepman KP, Smeele LE, van der Wal JE, Bezemer PD, van der Waal I.The possible premalignant character of oral lichen planus and oral lichenoid lesions: a prospective study. Oral Surg Oral Med Oral Pathol Oral Radiol Endod 2003;96:164-71.

4. Eisen D, Carozzo M, Bagán Sebastian JV, Thongprasom K. Number V Oral lichen planus: clinical features and management. Oral Dis 2005; 11:338-49.

5. WHO Collaborating Centro for Oral Precancerous Lesiones. Definition of keukoplakia and related 
lesions: an aid to studies on oral precancer. Oral Surg Oral Med Oral Pathol 1978; 46: 518-39.

6. van der Meij E, van der Waal I. Lack of clinicopathologic correlation in the diagnosis of oral lichen planus based on the presently available diagnostic criteria and suggestions for modifications. J Oral Pathol Med 2003;32:507-12.

7. Krutchkoff DJ, Eisemberg E. Lichenoid dysplasia: a distinct histopathologic entity. Oral Surg Oral Med Oral Pathol 1985 60:308-15.

8. Eisemberg E, Krutchkoff DJ. Lichenoid lesions of oral mucosa. Diagnostic criteria and their importance in the alleged relation-ship to oral cancer. Oral Surg Oral Med Oral Pathol 1992.

9. Lodi G, Scully C, Carozzo M, Griffiths M, Sugerman PB. Thogprasom K. Current controversies in oral lichen planus: Report of an international consensus meeting. Part 1. Viral infections and etiopathogenesis. Oral Surg Oral Med Oral Pathol Oral Radiol Endod 2005;100:40-51.

10. Lodi G, Scully C, Carozzo M, Griffiths M, Sugerman P.B. Thogprasom K. Current contro- versies in oral lichen planus:Report of an international consensus meeting. Part 2. Clinical management and malignant transformation. Oral Surg Oral Med Oral Pathol Oral Radiol Endod 2005;100:164-78.

11. Mignogna et al. Clinical guideliness in early detection of oral squamous cell carcinoma arising in oral lichen planus: a 5-year esperience. Oral Oncology 2001;37:262-7.

12. Mignogna, Fedele. Displasia/neoplasia surveillance in oral lichen planus patients: A description of clinical criteria adopted at a single centre and their impacto on prognosos Oral Oncology 2006;42:819-42.

13. Mattsson et al. Oral lichen planus and malignant transformation: Is a recall of patients justified? Crit Oral Biol Med 2002;13:390-6.

\section{CORRESPONDENCIA}

Rocio Cerero-Lapiedra

eMail: rcererol@infomed.es 